\title{
Protective effect of diminazene attenuates myocardial infarction in rats via increased inflammation and ACE2 activity
}

\author{
JUNJIANG CHEN $^{1}$, LIANQUN CUI ${ }^{2}$, JINGLIANG YUAN $^{3}$, SONGCUN ZHANG $^{3}$, \\ RONGMEI MA ${ }^{3}$, HONGJUN SANG ${ }^{3}$, QINGJIANG LIU ${ }^{3}$ and LIANFENG SHAN ${ }^{3}$ \\ ${ }^{1}$ Shandong University School of Medicine, Jinan, Shandong 250100; ${ }^{2}$ Department of Cardiology, \\ Shandong Provincial Hospital Affiliated to Shandong University, Jinan, Shandong 250021; \\ ${ }^{3}$ Shouguang People's Hospital, Shouguang, Shandong 262700, P.R. China
}

Received February 8, 2016; Accepted February 9, 2017

DOI: $10.3892 / \mathrm{mmr} .2017 .7152$

\begin{abstract}
The present study aimed to investigate whether diminazene attenuates myocardial infarction (MI) in rats. In addition, the present study investigated whether ACE2 signaling was involved in the effects of diminazene on protein function. A rat model of acute myocardial infarction (AMI) was established by occlusion of the left anterior descending coronary artery. The AMI model rats received intraperitoneal injections of diminazene $(5 \mathrm{mg} / \mathrm{kg} /$ day $)$ for 3 days. Treatment with diminazene significantly inhibited the expression of casein kinase and lactate dehydrogenase, and reduced infarct size in AMI rats. The findings indicated that diminazene significantly reduced the levels of inflammatory factors including tumor necrosis factor- $\alpha$ and interleukin-6, suppressed the protein expression of cytochrome c oxidase subunit 2 (COX-2) and inducible nitric oxide synthase (iNOS), and activated angiotensin-converting enzyme 2 (ACE2), angiotensin II receptor type 1 (AT1R) and MAS1 proto-oncogene, G protein-coupled receptor (MasR) protein expression in AMI model rats. In conclusion, the present study demonstrated that diminazene attenuated AMI in rats via suppression of inflammation, reduction of COX-2 and iNOS expression, and activation of the ACE2/AT1R/MasR signaling pathway.
\end{abstract}

\section{Introduction}

Cardiovascular disease has a negative effect on human health and wellbeing. In China, the number of patients with cardiovascular and cerebrovascular diseases has reached 230 million (1). It is estimated that $\sim 3$ million patients succumb to cardiovascular disease annually, which ranks in

Correspondence to: Dr Lianqun Cui, Department of Cardiology, Shandong Provincial Hospital Affiliated to Shandong University, Jinan, Shandong 250021, P.R. China

E-mail: lianquncuiyaf@163.com

Key words: diminazene, myocardial infarction, inflammation, ACE2, AT1R, MasR first place in causes of mortality, and the morbidity rate is continuously increasing (2). Additionally, acute myocardial infarction (AMI) threatens human health; AMI leads to the mortality of over half of the patients diagnosed. A previous study estimated that 17 million people worldwide succumb to cardiovascular disease annually (3). The prevalence of AMI is on the rise in China and is near the international average. Onset of acute AMI is sudden and the mortality rate is $~ 50 \%$ (4). Treatment of AMI has progressed; however, the risks of recurring myocardial infarction, congestive heart failure and other complications remain. It is estimated that $1 / 3$ of patients with MI may succumb within one year, particularly patients without congestive heart failure or left ventricular dysfunction (5). The fatality rate in hospitals reached $13.1 \%$, and was 5.5 times greater than in patients without congestive heart failure and left ventricular dysfunction (6). Cardiac failure and dysfunction of ventriculus sinister have already been identified as prognostic hazards of AMI (7).

Various cell types may contribute to the inflammatory response in congestive heart failure, including cardiac muscle, fibroblast, endothelial and infiltrative white blood cells. In addition, cardiac muscle cells are targets of the inflammatory response $(8,9)$.

Activated angiotensin-converting enzyme 2 (ACE2) is primarily expressed in the heart, kidneys, testes, main arteries, gastrointestinal tract, retinas and lungs (10). It is highly expressed in the heart and kidney tissues of humans and rodents. In the heart, ACE2 is primarily expressed in coronary vascular endothelial cells. The smooth muscle layer of coronary vessels exhibits a certain level of expression (11). The function of ACE2 is antagonistic to ACE: ACE converts angiotensin I (AngI) into AngII, a vasoconstrictor substance; and ACE2 converts AngII into theAng1-7 peptide, which is a strong vasodilator response substance. The function of ACE2 has two paths: i) it hydrolyzes AngI, and Ang1-9 is further hydrolyzed into Ang1-7; and ii) it hydrolyzes AngII directly to generate Ang1-7 (11). The efficiency of the latter path is 400-500 times greater than the first path. ACE2 in the renin-angiotensin (RAS) system may contribute to kidney disease, hypertension, heart failure, AMI and other pathological process. Long-term injections of Ang1-7 lead to the reduction of AngII expression levels in the hearts of rats (12). The expression level of ACE2 
was enhanced significantly, whereas AngII, ACE2 activity and AngII type 1 receptor (AT1R) concentrations did not differ. Therefore, Ang1-7 is a product after the reaction of ACE2 (11). ACE1 may enhance the expression levels of Ang1-7 and ACE2 and reduce the expression levels of AngII. Upregulation of ACE2 may be associated with a reduction inAng1-7 metabolism. AT1R antagonists enhance the expression levels of AngII and Ang1-7, and upregulate ACE2 expression (10). Additionally, ACE2 is effective where there is no AngII; therefore, there may be an independent pathway blocking AT1Rs.

A previous study revealed that diminazene (Fig. 1), alternatively known as Berenil and diminazene (13), may activate the ACE2/Ang1-7/MasR axis of the RAS system (14). Diminazene may be an activator of ACE2, as it has been demonstrated that it may improve the activity of ACE2 (15). Additionally, diminazene may have a protective role in pulmonary hypertension and cerebral arterial thrombosis via the regulation of the protective ACE2/Ang1-7/Mas-R axis. The aim of the present study was to evaluate the protective effect of diminazene on the attenuation of AMI in rats.

\section{Materials and methods}

AMI rat model. All experiments were performed in accordance with the Ethics Committee of Shandong University of Medical Sciences (Jinan, China). A total of 30 Sprague-Dawley rats (weight, 250-300 g; age 8-10 weeks) were obtained from the Animal Experimental Center of Shandong University and maintained at a room temperature $\left(23 \pm 2^{\circ} \mathrm{C}\right)$ with a 12/12 h light-dark cycle. Rats were randomly divided into the following 3 groups ( $n=10$ per group): i) Control group; ii) AMI model group; and iii) AMI + diminazene group, which received $5 \mathrm{mg} / \mathrm{kg} /$ day intraperitoneal injections of diminazene (Aburaihan Pharmaceutical Co., Tehran, Iran) for 3 days. Briefly, the rats were anesthetized using an intraperitoneal injection of ketamine $(80 \mathrm{mg} / \mathrm{kg})$ and xylazine $(5 \mathrm{mg} / \mathrm{kg}$; Sigma-Aldrich; Merck Millipore, Darmstadt, Germany). The AMI model was established by a silk suture (Huaiyin Medical Instruments Co., Ltd., Huaian, China) used to block the left anterior descending coronary artery. All rats were sacrificed by an intraperitoneal injection of $200 \mathrm{mg} / \mathrm{kg}$ pentobarbital (Sigma-Aldrich; Merck Millipore).

Laboratory analysis. Blood samples $(\sim 6 \mathrm{ml})$ were collected from the common carotid artery; serum was obtained following centrifugation at $2,000 \times \mathrm{g}$ for $10 \mathrm{~min}$ at $4^{\circ} \mathrm{C}$ and was subsequently stored at $-20^{\circ} \mathrm{C}$. Casein kinase $(\mathrm{CK}$; catalog no. A032), lactate dehydrogenase (LDH; catalog no. A020-2), tumor necrosis factor- $\alpha$ (TNF- $\alpha$; catalog no. H052) and interleukin (IL)-6 (catalog no. H007) were quantified using commercial ELISA kits obtained from Nanjing Jiancheng Bi oengineering Institute (Nanjing, China), following the manufacturer's protocol.

Western blot analysis. Rat hearts were excised and stored at $-80^{\circ} \mathrm{C}$. Rat heart tissue samples $(50 \mathrm{mg})$ were lysed with ice-cold lysis buffer containing phenylmethylsulfonyl fluoride (Beyotime Institute of Biotechnology, Inc., Haimen, China). Protein concentrations were measured using a Bicinchoninic Protein Assay kit (Beyotime Institute of Biotechnology, Inc.). A<smiles>[R2]C(=O)NCC(=O)O</smiles>

Figure 1. Chemical structure of diminazene.

total of $50 \mu \mathrm{g}$ protein was loaded onto $10-12 \%$ gels, subjected to SDS-PAGE and transferred onto polyvinylidene difluoride membranes (EMD Millipore, Billerica, MA, USA). The membranes were blocked with Tris-buffered saline containing $5 \%$ non-fat milk and $0.05 \%$ Tween-20, and incubated with the following primary antibodies (Santa Cruz Biotechnology, Inc.; Dallas, TX, USA): Cytochrome c oxidase subunit 2 (COX-2; 1:300; catalog no. sc-7951), inducible nitric oxide synthase (iNOS; 1:300; catalog no. sc-649), ACE2 (1:300; catalog no. sc-20998), AT1R (1:300; catalog no. sc-134652), MAS1 proto-oncogene, G protein-coupled receptor (MasR; 1:300; catalog no. sc-32889) or GAPDH (1:300; catalog no. sc-367714) at $4^{\circ} \mathrm{C}$ overnight. Subsequently, membranes were washed with tris-buffered saline and Tween-20 and incubated with a goat anti-rabbit secondary antibody (1:10,000; catalog no. sc-2004; Santa Cruz Biotechnology, Inc.) at $37^{\circ} \mathrm{C}$ for $1 \mathrm{~h}$. Membranes were visualized using an Enhanced Chemiluminescence Detection kit (GE Healthcare Life Sciences, Shanghai, China) and quantified using a ScanJet 4C flatbed scanner (Hewlett-Packard, Palo Alto, CA, USA) with ImageJ software version 1.52 (National Institutes of Health, Bethesda, MD, USA).

Statistical analysis. Data are expressed as the mean \pm standard error of the mean. The results were analyzed using SPSS software version 19.0 (IBM SPSS, Armonk, NY, USA). Student's t-test was used for comparison of differences between the groups. $\mathrm{P}<0.05$ was considered to indicate a statistically significant difference.

\section{Results}

Protective effect of diminazene attenuates $C K$ and LDH levels in the AMI rat model. Following the establishment of the AMI model, the levels of CK and LDH in serum of rats were quantified. The findings of the present study indicated that the levels of $\mathrm{CK}$ and $\mathrm{LDH}$ in AMI rats were significantly higher compared with the control group (Fig. 2). Treatment with diminazene significantly reduced CK and LDH levels in AMI rats compared with the untreated AMI model group (Fig. 2).

Protective effect of diminazene attenuates infarct size in the AMI rat model. The present study investigated the effect of diminazene on infarct size in AMI rats. Infarct size was significantly increased in AMI rats compared with the control group (Fig. 3). However, treatment with diminazene significantly reduced infarct size in AMI rats, compared with the untreated AMI model group (Fig. 3).

Protective effect of diminazene attenuates inflammation factors in the AMI rat model. The protective effect of diminazene on the expression of inflammatory factors, including TNF- $\alpha$ and IL-6, in AMI rats was determined using 
A

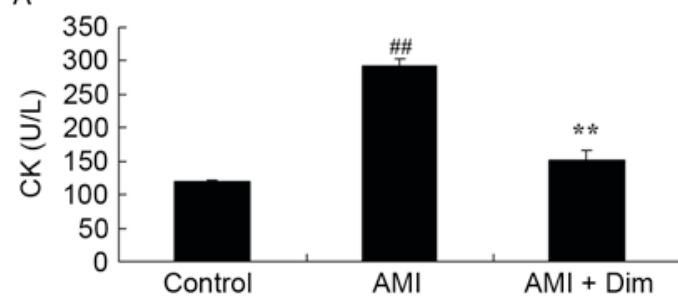

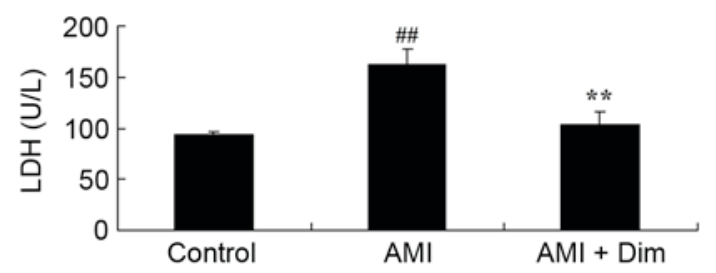

Figure 2. Protective effect of diminazene attenuated (A) CK and (B) LDH levels in AMI rat model. ${ }^{\# \#} \mathrm{P}<0.01$ vs. control group; ${ }^{* *} \mathrm{P}<0.01$ vs. AMI model group. AMI, acute myocardial infraction; CK, casein kinase; Dim, diminazene; LDH, lactate dehydrogenase.

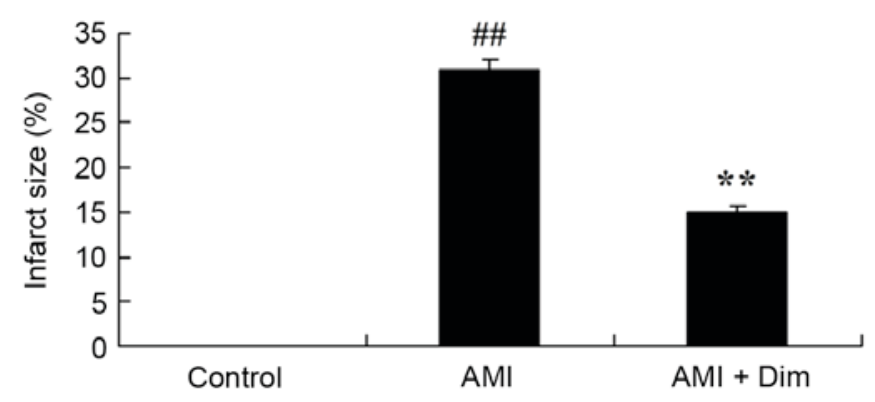

Figure 3. Protective effect of diminazene reduced infarct size in the AMI rat model. Control, control group; ${ }^{\# \#} \mathrm{P}<0.01$ vs. control group; ${ }^{* *} \mathrm{P}<0.01$ vs. AMI model group. AMI, acute myocardial infraction; Dim, diminazene.

commercially available ELISA kits. There was a significant increase in TNF- $\alpha$ and IL-6 levels in the AMI rat model group compared with the control group (Fig. 4). Diminazene treatment significantly reduced the serum concentration of TNF- $\alpha$ and IL-6 in AMI rats compared with the untreated AMI model group (Fig. 4).

Protective effect of diminazene attenuated COX-2 and iNOS protein expression in AMI rat model. Western blot analysis was used to determine COX-2 and iNOS protein expression in AMI model rat. The results showed that COX-2 and iNOS protein expression was significantly activated by AMI in rat, compared with control group (Fig. 5). Treatment with diminazene significantly suppressed the AMI-induced COX-2 and iNOS protein expression in rat, compared with AMI model group (Fig. 5).

Protective effect of diminazene activates ACE2, AT1R and MasR protein expression in the AMI rat model. Western blotting was used to determine the MasR protein expression in AMI rats. ACE2, AT1R and MasR protein expression in the AMI group was significantly reduced compared with the control group (Fig. 6). Treatment with diminazene significantly increased ACE2, AT1R and MasR protein expression levels in AMI rats compared with the untreated AMI group (Fig. 6).

\section{Discussion}

AMI is myocardial ischemic necrosis that occurs via an acute reduction or interruption of blood supply due to lesions in the coronary artery, which leads to cardiac ischemia and results in myocardial necrosis (2). Manifestation of AMI usually involves retrosternal pain, fever, increased white blood cells and increased red blood cell sedimentation rate (16). In addition, AMI may be associated with arrhythmia, shock or cardiac failure, and may endanger patient survival. In patients with irreversible damage to cardiac muscle cells in ventriculus sinister, $>40 \%$ will develop left-sided heart failure, acute pulmonary edema or cardiogenic shock (17). AMI occurs due to acute occlusion and interruption of blood flow through the coronary artery, resulting in myocardial necrosis due to continuous ischemia and anoxia in the cardiac muscle cells (3). In the present study, diminazene significantly decreased the level of CK and LDH expression, and reduced infarct size in AMI rats. These findings suggested that diminazene may suppress myocardial infarction in vitro.

AMI frequently results in chronic heart failure and may lead to an inflammatory response of the heart. A previous study revealed that there was an increase in the expression levels of inflammatory factors in the heart ischemic region and far-end region following myocardial infarction $(18,19)$. Inflammation occurs due to myocardial damage, and blocked blood vessels leads to a stress reaction that results in inflammation. This process is accompanied by infiltration of inflammatory cells (20). Notably the far-end region is maintained in an inflammatory state for up to 7 weeks following the occurrence of AMI. The present study revealed that diminazene treatment significantly reduced the activity of TNF- $\alpha$ and IL-6 in the serum of AMI rats. Kuriakose et al demonstrated that diminazene suppressed inflammatory responses to Trypanosoma congolense infection (21).

ACE degrades Ang1-7 and inhibits the activity of ACE to increase Ang1-7 expression levels and restrain AT1R, and leads to the accumulation of AngII, which results in degradation of productive Ang1-7 (12). Degradation of Ang1-7 requires the amino terminal of ACE. Ang1-7 may stimulate the circulatory system to release bradykinin, prostaglandin and endothelial tissue-derived nitric oxide (NO), to increase natriuresis and to reduce sodium-potassium-ATP enzyme activity in kidney tubules, to effectively depressurize blood vessels (22). Following myocardial infarction, long-term injections of Ang1-7 may improve cardiac function, coronary perfusion and vascular endothelial cell of aorta, and protect cardiac muscle from ischemia-reperfusion injury (23). The present study demonstrated that diminazene significantly reduced the AMI-induced protein expression levels of COX-2 and iNOS in AMI rats. Zheng et al (24) revealed that diminazene diaceturate reduced inflammation in endotoxin-induced 
A

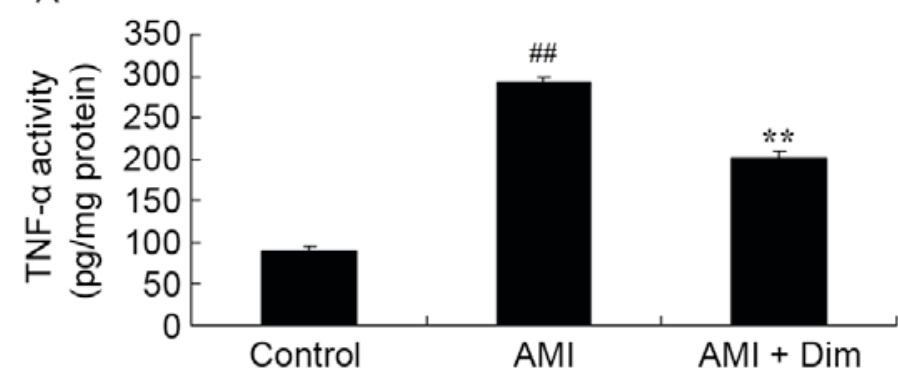

B

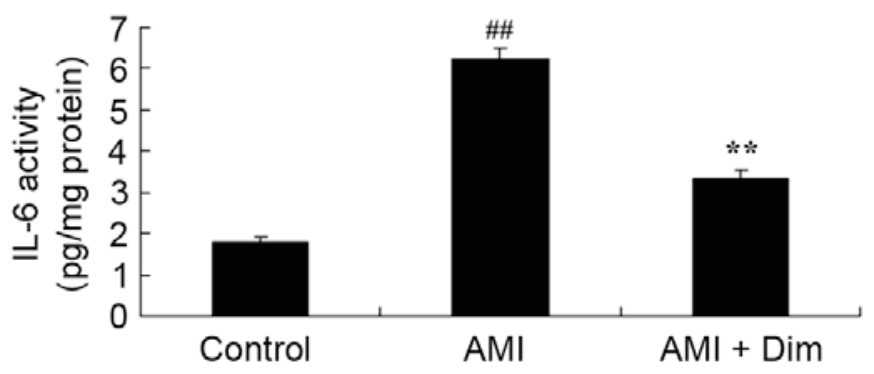

Figure 4. Protective effect of diminazene attenuated inflammation factors in the AMI rat model. Protective effect of diminazene attenuates the activity of (A) TNF- $\alpha$ and (B) IL-6 in serum of AMI rats. ${ }^{\# \# ~} \mathrm{P}<0.01$ vs. control group; ${ }^{* *} \mathrm{P}<0.01 \mathrm{vs}$. AMI model group. AMI, acute myocardial infraction; Dim, diminazene; IL-6, interleukin-6; TNF- $\alpha$, tumor necrosis factor- $\alpha$.

\section{A}

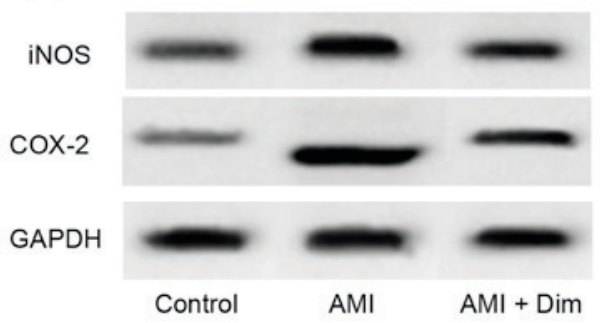

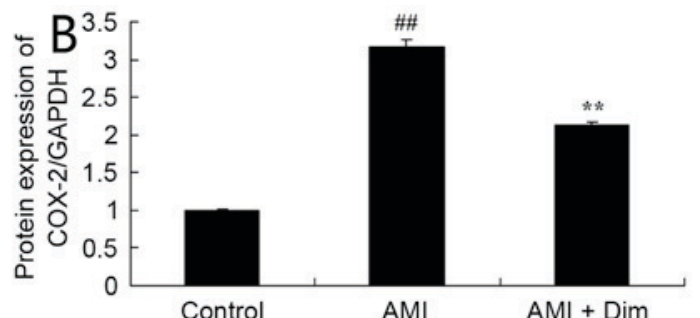

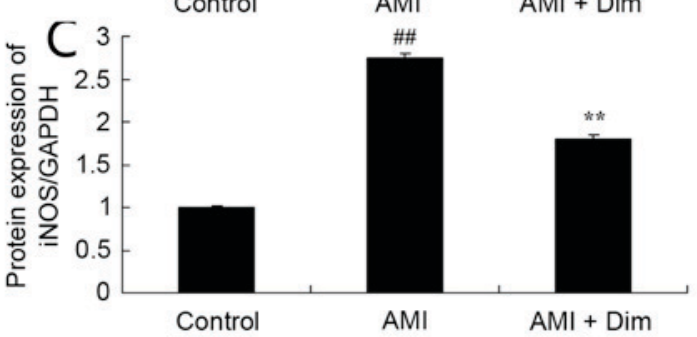

Figure 5. Protective effect of diminazene attenuated COX-2 and iNOS protein expression in AMI rat model. Effect of diminazene on COX-2 and iNOS protein expression was determined using (A) western blot analysis and quantification of (B) COX-2 and (C) iNOS protein expression in AMI rat model. ${ }^{\# \#} \mathrm{P}<0.01$ vs. control group; ${ }^{* *} \mathrm{P}<0.01$ vs. AMI model group. AMI, acute myocardial infraction; Dim, diminazene; COX-2, cytochrome c oxidase subunit 2.
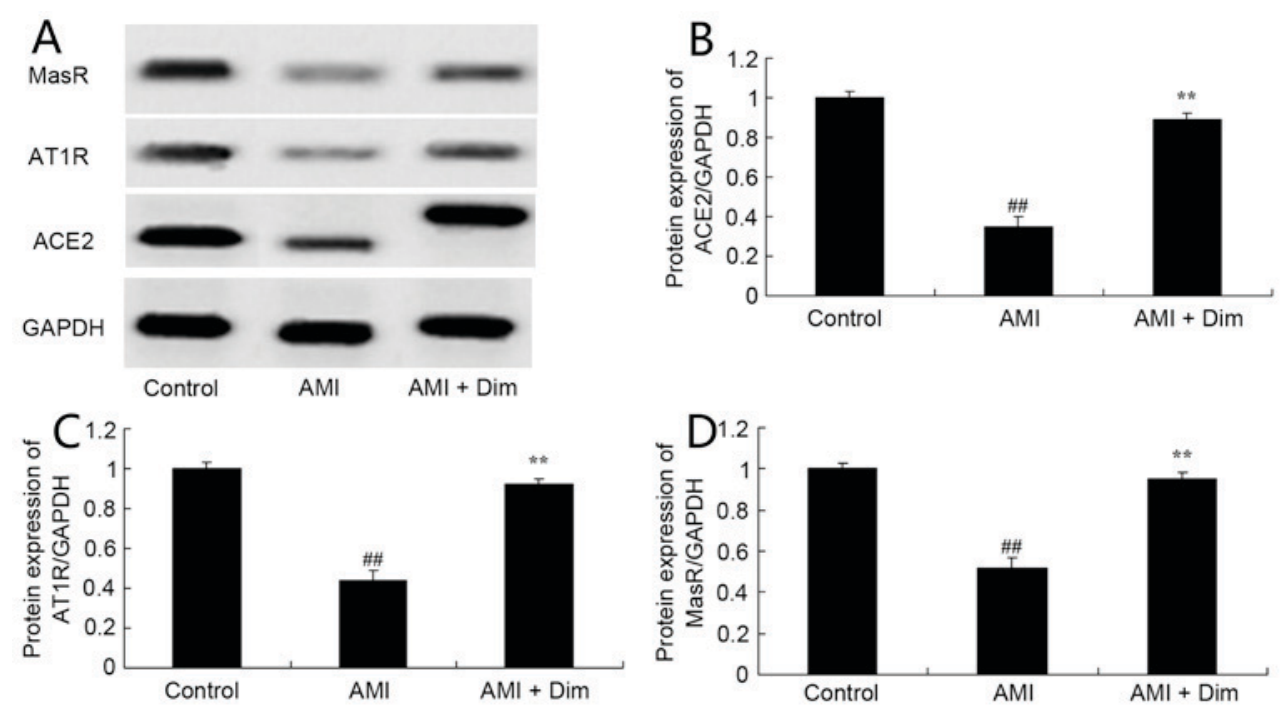

Figure 6. Protective effect of diminazene activated ACE2, AT1Rand MasR protein expression in the AMI rat model. Effect of diminazene on ACE2, AT1R and MasR protein expression was determined using (A) western blot analysis and quantification of (B) ACE2, (C) AT1R and (D) MasR protein expression in the AMI rat model. ${ }^{\# \#} \mathrm{P}<0.01$ vs. control group; ${ }^{* *} \mathrm{P}<0.01$ vs. AMI model group. AMI, acute myocardial infraction; Dim, diminazene; MasR, MAS1 proto-oncogene, $\mathrm{G}$ protein-coupled receptor. 
uveitis via reduction of COX-2 and iNOS. These findings indicated that the protective effect of diminazene on AMI was associated with COX-2 and iNOS expression levels.

A previous study revealed that the function of the ACE2/Ang1-7/MasR axis is to relax blood vessels, reduce proliferation of cardiac muscle cells, reverse ventricular remodeling and improve cardiac function, in addition to having anti-inflammatory, anti-coagulant and anti-arrhythmic effects (25). The ACE2 channel regulates cardiac remodeling following AMI, and it is crucial for recovery (26). AngII expression in cardiac muscle tissue and the infarction region following AMI is enhanced. The levels of ACE2, Ang1-7 and MasR were increased slightly following AMI (27). Another study demonstrated the ACE2, Ang1-7 and MasR protein expression levels in the cardiac muscle tissue of AMI rats, and the mRNA expression level of AngII were enhanced compared with control rats, confirming RAS activation in the heart following AMI (26). The present study revealed that treatment with diminazene resulted in the reduction of ACE2, AT1R and MasR protein expression levels in AMI rats. Malek et al (15) reported that diminazene may protect the male kidney from renal ischemic-reperfusion injury via the elevation of ACE2, AT1R and MasR protein expression levels. The findings of the present study indicate that the regulation of ACE2/AT1R/MasR axis may influence the protective effect of diminazene in AMI.

In conclusion, the present study demonstrated that diminazene significantly reduced CK and LDH activity levels, and reduced infarct size in vitro following induction of AMI in rats. In addition, this effect was partially mediated by the suppression of inflammation, reduced COX-2 and iNOS levels, and activation of the ACE2/AT1R/MasR axis. These findings suggest that diminazene may protect against AMI via the ACE2/AT1R/MasR axis and may be a novel therapeutic agent for the treatment of AMI.

\section{References}

1. Planer D, Smits PC, Kereiakes DJ, Kedhi E, Fahy M, Xu K, Serruys PW and Stone GW: Comparison of everolimus- and paclitaxel-eluting stents in patients with acute and stable coronary syndromes: Pooled results from the SPIRIT (A Clinical Evaluation of the XIENCE V Everolimus Eluting Coronary Stent System) and COMPARE (A trial of everolimus-eluting stents and paclitaxel-eluting stents for coronary revascularization in daily practice) trials. JACC Cardiovasc Interv 4: 1104-1115, 2011.

2. Wang TY, Henry TD, McCoy LA, Berger PB, Cohen DJ, Effron MB, Zettler M, Baker BA, Messenger JC and Peterson ED: Contemporary use of platelet function and pharmacogenomic testing among patients with acute myocardial infarction undergoing percutaneous coronary intervention in the United States. Am Heart J 170: 706-714, 2015.

3. Ellis SG, Tendera M, de Belder MA, van Boven AJ, Widimsky P, Janssens L, Andersen HR, Betriu A, Savonitto S, Adamus J, et al: Facilitated PCI in patients with ST-elevation myocardial infarction. N Engl J Med 358: 2205-2217, 2008.

4. Zhang Z, Li K and Tian J: Efficacy and safety outcomes of fractional flow reserve in guiding clinical therapy of non-ST-segment elevation myocardial infarction compared with angiography alone in elderly Chinese patients. Clin Interv Aging 11: 1751-1754, 2016.

5. APEX AMI Investigators, Armstrong PW, Granger CB, Adams PX, Hamm C, Holmes D Jr, O'Neill WW, Todaro TG, Vahanian A and Van de Werf F: Pexelizumab for acute ST-elevation myocardial infarction in patients undergoing primary percutaneous coronary intervention: A randomized controlled trial. JAMA 297: 43-51, 2007.
6. Hausenloy DJ, Kharbanda R, Rahbek Schmidt M, Møller UK, Ravkilde J, Okkels Jensen L, Engstrøm T, Garcia Ruiz JM, Radovanovic N, Christensen EF, et al: Effect of remote ischaemic conditioning on clinical outcomes in patients presenting with an ST-segment elevation myocardial infarction undergoing primary percutaneous coronary intervention. Eur Heart J 36: 1846-1848, 2015.

7. Gurfinkel EP, de la Fuente RL, Mendiz O and Mautner B: Influenza vaccine pilot study in acute coronary syndromes and planned percutaneous coronary interventions: The FLU Vaccination Acute Coronary Syndromes (FLUVACS) Study. Circulation 105: 2143-2147, 2002.

8. Halvorsen S, Seljeflot I, Weiss T, Bøhmer E and Arnesen H: Inflammatory and thrombotic markers in patients with ST-elevation myocardial infarction treated with thrombolysis and early PCI: A NORDISTEMI substudy. Thromb Res 130: 495-500, 2012.

9. Abbate A, Van Tassell BW, Christopher S, Abouzaki NA, Sonnino C, Oddi C, Carbone S, Melchior RD, Gambill ML, Roberts CS, et al: Effects of Prolastin C (Plasma-Derived Alpha-1 Antitrypsin) on the acute inflammatory response in patients with ST-segment elevation myocardial infarction (from the VCU-alpha 1-RT pilot study). Am J Cardiol 115: 8-12, 2015.

10. Ortiz-Perez JT, Riera M, Bosch X, De Caralt TM, Perea RJ, Pascual $J$ and Soler MJ: Role of circulating angiotensin converting enzyme 2 in left ventricular remodeling following myocardial infarction: A prospective controlled study. PLoS One 8: e61695, 2013

11. Mendoza-Torres E, Oyarzún A, Mondaca-Ruff D, Azocar A, Castro PF, Jalil JE, Chiong M, Lavandero S and Ocaranza MP: ACE2 and vasoactive peptides: Novel players in cardiovascular/renal remodeling and hypertension. Ther Adv Cardiovasc Dis 9: 217-237, 2015.

12. Bennion DM, Haltigan E, Regenhardt RW, Steckelings UM and Sumners C: Neuroprotective mechanisms of the ACE2-angiotensin-(1-7)-Mas axis in stroke. Curr Hypertens Rep 17: 3, 2015.

13. Peregrine AS and Mamman M: Pharmacology of diminazene: A review. Acta Trop 54: 185-203, 1993.

14. Kuriakose S and Uzonna JE: Diminazene aceturate (Berenil), a new use for an old compound? Int Immunopharmacol 21: 342-345, 2014.

15. Malek M and Nematbakhsh M: The preventive effects of diminazene aceturate in renal ischemia/reperfusion injury in male and female rats. Adv Prev Med 2014: 740647, 2014.

16. Guagliumi G, Sirbu V, Bezerra H, Biondi-Zoccai G, Fiocca L, Musumeci G, Matiashvili A, Lortkipanidze N, Tahara S, Valsecchi $O$ and Costa M: Strut coverage and vessel wall response to zotarolimus-eluting and bare-metal stents implanted in patients with ST-segment elevation myocardial infarction: the OCTAMI (Optical Coherence Tomography in Acute Myocardial Infarction) study. JACC Cardiovasc Interv 3: 680-687, 2010.

17. Malliaras K, Makkar RR, Smith RR, Cheng K, Wu E, Bonow RO, Marbán L, Mendizabal A, Cingolani E, Johnston PV, et al: Intracoronary cardiosphere-derived cells after myocardial infarction: Evidence of therapeutic regeneration in the final 1-year results of the CADUCEUS trial (Cardiosphere-Derived autologous stem Cells to reverse ventricular dysfunction). J Am Coll Cardiol 63: 110-122, 2014

18. Liu ML, Nagai T, Tokunaga M, Iwanaga K, Matsuura K, Takahashi T, Kanda M, Kondo N, Naito AT, Komuro I and Kobayashi Y: Anti-inflammatory peptides from cardiac progenitors ameliorate dysfunction after myocardial infarction. J Am Heart Assoc 3: e001101, 2014.

19. Yang K, Zhang Y, Xu C, Li X and Li D: mTORC1 signaling is crucial for regulatory $\mathrm{T}$ cells to suppress macrophage-mediated inflammatory response after acute myocardial infarction. Immunol Cell Biol 94: 274-284, 2016.

20. Aggarwal A, Atreja A, Kapadia S, Lopez R and Achkar JP: Conventional risk factors and cardiovascular outcomes of patients with inflammatory bowel disease with confirmed coronary artery disease. Inflamm Bowel Dis 20: 1593-1601, 2014.

21. Kuriakose S, Muleme HM, Onyilagha C, Singh R, Jia P and Uzonna JE: Diminazene aceturate (Berenil) modulates the host cellular and inflammatory responses to Trypanosoma congolense infection. PLoS One 7: e48696, 2012. 
22. Pernomian L, Pernomian L and Baraldi Araújo Restini C: Counter-regulatory effects played by the ACE-Ang II-AT1 and ACE2-Ang-(1-7)-Mas axes on the reactive oxygen species-mediated control of vascular function: perspectives to pharmacological approaches in controlling vascular complications. Vasa 43: 404-414, 2014.

23. Patel VB,Parajuli N and Oudit GY: Role of angiotensin-converting enzyme 2 (ACE2) in diabetic cardiovascular complications. Clin Sci (Lond) 126: 471-482, 2014.

24. Zheng C, Lei C, Chen Z, Zheng S, Yang H, Qiu Y and Lei B: Topical administration of diminazene aceturate decreases inflammation in endotoxin-induced uveitis. Mol Vis 21: 403-411, 2015.
25. Li Y, Cao Y, Zeng Z, Liang M, Xue Y, Xi C, Zhou M and Jiang W: Angiotensin-converting enzyme 2/angiotensin-(1-7)/Mas axis prevents lipopolysaccharide-induced apoptosis of pulmonary microvascular endothelial cells by inhibiting JNK/NF-kB pathways. Sci Rep 5: 8209, 2015.

26. Oudit GY and Penninger JM: Recombinant human angiotensin-converting enzyme 2 as a new renin-angiotensin system peptidase for heart failure therapy. Curr Heart Fail Rep 8: 176-183, 2011.

27. Wang W, Bodiga S, Das SK, Lo J, Patel V and Oudit GY: Role of ACE2 in diastolic and systolic heart failure. Heart Fail Rev 17: 683-691, 2012. 